\title{
DNA methylation markers of surfactant proteins in lung cancer
}

\author{
ZHENWU LIN $^{1}$, NEAL J. THOMAS ${ }^{2,3}$, MARINA BIBIKOVA ${ }^{5}$, CAROLA SEIFART ${ }^{6}$, YUNHUA WANG ${ }^{1}$, \\ XIAOXUAN GUO ${ }^{1}$, GUIRONG WANG ${ }^{1}$, EKKEHARD VOLLMER ${ }^{7}$, TORSTEN GOLDMANN ${ }^{7}$, \\ ELIZA WICKHAM GARCIA ${ }^{5}$, LIXIN ZHOU ${ }^{5}$, JIAN-BING FAN ${ }^{5}$ and JOANNA FLOROS ${ }^{1,2,4}$
}

\begin{abstract}
Departments of ${ }^{1}$ Cellular and Molecular Physiology, ${ }^{2}$ Pediatrics, ${ }^{3}$ Health Evaluation Sciences, and ${ }^{4}$ Obstetrics and Gynecology, Pennsylvania State University College of Medicine, Hershey, PA 17033; ${ }^{5}$ Illumina, Inc., San Diego, CA 92121, USA; ${ }^{6}$ Department of Internal Medicine, Division of Respiratory Medicine, University of Giessen and Marburg, 35043 Marburg; ${ }^{7}$ Clinical and Experimental Pathology, Research Center Borstel, Parkallee, 23845 Borstel, Germany
\end{abstract}

Received February 26, 2007; Accepted April 17, 2007

\begin{abstract}
Surfactant proteins play important roles in lung surfactant function and innate immunity. The DNA methylation state of $11 \mathrm{CpG}$ sites of surfactant protein (SP)-A1, -B, -C, and $-\mathrm{D}$ was determined using universal bead arrays. A total of 90 cancerous and non-cancerous tissues from 23 patients with adenocarcinoma and 22 with squamous cell carcinoma were studied. These were divided into a training set and a testing set. The results indicate that DNA methylation profiling of these $\mathrm{CpGs}$ is associated with lung cancer. Four $\mathrm{CpG}$ sites, SP-A1_370, SP-A1_1080, SP-D_1170, and SP-D_1370, were hypomethylated in cancer and were significantly associated with both adenocarcinoma and squamous cell carcinoma, indicating that they have the potential to be used as biomarkers for lung cancer diagnosis and treatment. Normal lung tissues with a higher level of unmethylated SP-A1_1468 and SPD_1170 CpG exhibited a higher level of SP-A1 and SP-D gene transcripts indicating that $\mathrm{CpG}$ methylation may play a role in gene expression. When the non-cancerous tissues were compared to cancerous tissues in patients with adenocarcinoma, the methylation profile results of these 46 samples (23 cancerous and 23 non-cancerous) could be clustered into 4 groups by agglomerative nesting. The percentage of tumor samples in each group was $0,58,91$, and 100 , respectively. A similar pattern was observed in squamous cell carcinoma patients. We speculate that SP-A1 and SP-D are subject to methylation/demethylation regulatory mechanisms and are involved in lung cancer pathogenesis by virtue of their function in innate host defense and/or regulation of inflammation.
\end{abstract}

Correspondence to: Professor Joanna Floros, Department of Cellular and Molecular Physiology, Pennsylvania State University College of Medicine, 500 University Drive, Hershey, PA 17033, USA

E-mail: jfloros@psu.edu or jxf19@psu.edu

Dr Jian-Bing Fan, Illumina, Inc., San Diego, CA 92121, USA

E-mail: jfan@illumina.com

Key words: lung cancer, DNA methylation, microarray, surfactant proteins, epigenetics

\section{Introduction}

DNA methylation is an important epigenetic alteration in the mammalian genome. DNA methylation of $\mathrm{CpG}$ islands in the promoter region of genes has been recognized as a factor for the regulation of gene expression. DNA methylation has been found to be an important player in development and differentiation of many human diseases $(1,2)$.

DNA methylation in cancer has been widely studied. Although there appears to be a global hypomethylation in the genome of the cancer cell, hypermethylation of tumor suppression genes also has been reported in many types of cancers (3-5). DNA methylation can cause gene silencing as well as decreased expression of cancer suppressor genes $(6,7)$. Several genes with an altered methylation pattern have been observed in lung cancer, including p16, CDKN2A, RARß, RASSF1A, Adenomatous Polyposis Coli, and DRP kinase $(8,9)$.

DNA methylation/demethylation is also affected by environmental factors, such as diet, air pollution, and smoking $(10,11)$. The human lung is one of the organs that faces these challenges directly, and therefore it is biologically plausible to speculate that pulmonary cells may be at increased risk of developing altered DNA methylation patterns due to these environmental stressors. Innate immunity provides the first line of host defense against such challenges. Although an acute inflammatory response is a healing event, chronic inflammation is a common hallmark of many lung diseases, and inflammation is a critical component of tumor progression (12). The tumor microenvironment appears to be largely orchestrated by inflammatory cells as many tumors arise from sites of inflammation. Tumor cells have co-opted some of the signaling molecules of innate immunity systems (13), such as the nuclear transcription factor $\kappa \mathrm{B}(\mathrm{NF}-\kappa \mathrm{B})$. NF- $\kappa \mathrm{B}$ is a hallmark of inflammation, and is abnormally active in some cancers. NF- $\mathrm{KB}$ activity is involved in both early and late events in the development of cancer and may lead to inhibition of programmed cell death (14).

The surfactant proteins (SP-A, -B, -C, and -D) are important for normal lung function $(15,16)$ and SP-B is essential for life $(17,18)$. SP-B and SP-C play a key role in the surface tension lowering activity of surfactant, and SP-A and SP-D are 
Table I. Lung cancer samples used in this study.

\begin{tabular}{lccccc}
\hline & \multicolumn{2}{c}{ Adenocarcinoma } & & \multicolumn{2}{c}{ Squamous cell carcinoma } \\
\cline { 2 - 3 } \cline { 5 - 6 } \cline { 5 - 6 } & Tumor & Matched normal & & Tumor & Matched normal \\
\hline Training set & 11 & 11 & & 14 & 14 \\
Testing set & 12 & 12 & & 8 & 8 \\
Total & 23 & 23 & & 22 & 22 \\
\hline
\end{tabular}

important for the host defense function of the lung. Genetic variants of these genes have been associated with inflammatory lung diseases, such as respiratory distress syndrome (RDS), acute RDS (ARDS), chronic obstructive pulmonary disease (COPD), and lung cancer (19-24).

We hypothesized that one mechanism by which expression of surfactant protein genes is regulated is by DNA methylation/ demethylation of their promoter region, and via this altered expression the surfactant proteins contribute to lung inflammatory diseases and lung cancer. We have previously described a high-throughput DNA methylation profiling method using a universal bead array with normal and adenocarcinoma lung tissues (25). In the present report, we focus on the methylation pattern of the surfactant genes (SP-A1, SP-B, SP-C, SP-D) in lung cancer, and report that the methylation status of SP-A1 and SP-D is associated with lung adenocarcinoma and squamous cell cancer. We further studied the effect of SP-A1 and SP-D methylation on gene expression.

\section{Materials and methods}

Lung tissues. For the bead array methylation analysis, lung tissues were collected during surgery for tumor removal, and were classified by clinical pathologists as cancerous. Tissue adjacent to the cancerous tissue, but without histological features of cancer, was noted as normal (non-cancerous). The tissues were collected under a protocol approved by the Human Subjects Protection Offices of The Pennsylvania State University College of Medicine, USA, and The University of Research Center Borstel, Germany. Upon resection, the samples were classified into cancerous and non-cancerous, frozen, and stored at $-80^{\circ} \mathrm{C}$. A total of 90 samples (45 cancerous and 45 non-cancerous) from 23 adenocarcinoma and 22 squamous cell carcinoma (SCC) patients were collected for this study. The training set samples from 11 adenocarcinoma and 14 SCC patients were obtained from Philips-University of Marburg, Germany, and the testing set samples from 12 adenocarcinoma and 8 SCC patients were obtained from The Penn State Cancer Institute Tumor Bank (Table I). Normal lung tissue $(\mathrm{n}=10)$ was obtained from the Gift of Life Donor Program (Philadelphia, PA) and was used for SP-A gene-specific PCR amplification and gene expression portions of this project.

DNA samples for methylation analysis. The lung tissues were pulverized under liquid nitrogen and genomic DNA was isolated from the tissue powder by QIAamp DNA mini kit (Qiagen Inc. Valencia, CA) according to the manufacturer's instructions. The DNA was eluted from the column with $\mathrm{dH}_{2} \mathrm{O}$, and stored at $-80^{\circ} \mathrm{C}$ until use. DNA concentration was measured by Quant-iT ${ }^{\text {TM }}$ PicoGreen dsDNA Assay kit (Molecular Probe, Eugene, OR).

For reference, the following DNAs were used. DNA from lung cancer cell lines NCI-H69 (HTB-119D), NCI-H526 (CRL-5807D), NCI-H358 (CRL-5811D), NCI-H1299 (CRL5803D), NCI-H1395 (CRL-5868D), and NCI-H2126 (CCL256D) was purchased from ATCC (Manassas, VA). DNA samples from normal lung were purchased from Clinomics Biosciences (Frederick, MD). DNA samples NA06999, NA07033, NA10923, and NA10924 were purchased from the Coriell Institute for Medical Research (Camden, NJ).

$C p G$ sites of surfactant protein genes for analysis. A total of $11 \mathrm{CpG}$ sites in the 5'-flanking or promoter region of human surfactant protein genes were studied. Among these, 3 were from the SP-A1 gene, 3 were from the SP-B gene, 3 were from the SP-C gene, and 2 were from the SP-D gene. All the $\mathrm{CpG}$ sites and the flanking sequences are listed in Table II. The SP-A2 gene was not included because the sequence of SP-A2 was not available from the DNA database at the time of experimentation.

Bisulfite conversion of DNA and methylation assay. The EZ DNA methylation kit (for micro-array assay) or the methylationGold kit (for PCR-based cRFLP method) (Zymo Research, Orange, CA) was used for bisulfite conversion of all DNA samples, according to the manufacturer's recommendations. One microgram of genomic DNA was used for each conversion reaction. Bisulfite-converted genomic DNA from one conversion was then used for up to 5 array experiments. After bisulfite conversion of genomic DNA, the remaining assay steps were identical to the GoldenGate ${ }^{\mathrm{TM}}$ genotyping assay (26), using Illumina-supplied reagents and conditions (BeadLab User's Manual, Illumina, San Diego, CA). Single-stranded PCR products were prepared by denaturation, then hybridized to a Sentrix ${ }^{\circledR}$ array matrix. The array hybridization was conducted under a temperature gradient program, and arrays were imaged using a BeadArray Reader 1000 scanner. Image processing and intensity data extraction software were as described previously (27). DNA methylation assays were performed by Illumina BeadArray $^{\mathrm{TM}}$ technology and methylation data analysis was described previously (25).

PCR-based cRFLP method for analysis of CpG methylation of $S P-A 1 \_1468$ and $S P-D \_1170$. In the bisulfite converted genomic DNA, all unmethylated cytosines (C) were converted to uracil, while methylated $\mathrm{Cs}$ remained unchanged. The hybridization behavior of uracil is similar to that of $\mathrm{T}$, therefore, the unmethylated $\mathrm{C}$ and methylated $\mathrm{C}$ can be considered as a T/C polymorphism. The SNP T/C can be changed to a restriction enzyme recognition site by, in most cases, one mismatched nucleotide in a PCR primer. The T/C can then be analyzed by converted RFLP (cRFLP) methods. The target sequence was first amplified by PCR with primers 1494/1502 for SP-A1_1468 and $1517 / 1520$ for SP-D_1170 at $95^{\circ} \mathrm{C}$ for $2 \mathrm{~min}, 35$ cycles of $95^{\circ} \mathrm{C}$ for $30 \mathrm{sec}, 55^{\circ} \mathrm{C}$ for $1 \mathrm{~min}$, and $72^{\circ} \mathrm{C}$ for $1 \mathrm{~min}$, and $72^{\circ} \mathrm{C}$ for $4 \mathrm{~min}$. Then the PCR products were used as a template for nested PCR with 1501/1508 for SP-A1_1468 and 1518/1519 for SP-D_1170 at $95^{\circ} \mathrm{C}$ for $2 \mathrm{~min}$, 
Table II. Targeting CpG sites of SPs and their flanking sequences.

\begin{tabular}{llrrl}
\hline CpG site & Strand & Nt $^{\mathrm{a}}$ & & Target sequence $^{\mathrm{b}}$ \\
\hline SP-A1_370 & Reverse & 45 & 347 & GTGTGCCTTTCCCCTAAAGTCACGCCCTGCCCTGCCAACCACAAG 391 \\
SP-A1_1080 & Forward & 48 & 1057 & TGGGGCTCATGGCTGAGCCAGGTCGCAGGACAGACAAGTTGGCCTGGA 1104 \\
SP-A1_1468 & Reverse & 53 & 1444 & AGCCCCCACATCTATAAATGCTGCGTCTACCTTACCCTCTGACTTGGAGGCAG 1496 \\
SP-B_551 & Reverse & 52 & 526 & TTCATCATGGTACTAATTCCTGCCCGTCCACCCACAAAAGCACTGTAGTGCT 577 \\
SP-B_812 & Reverse & 49 & 786 & GGACAGTTTCTTTCTGCTGAACCATCGCAGCTATGCCCCAGCCCCTACC 834 \\
SP-B_1448 & Reverse & 43 & 1429 & ACTCCTACAGAGCCCCCACGCCCCGCCCAGCTATAAGGGGCCA 1471 \\
SP-C_358 & Forward & 47 & 335 & AAAAGGATAGGAGGTTAAGGGAGCGAGCCCAGGCCTGGACTCTGCCA 381 \\
SP-C_1218 & Forward & 53 & 1191 & GGCAGGTGCCAGCAAGGAAGGCAGGCACGCCAGGAAGACACCCATGGTGAGAA 1243 \\
SP-C_1367 & Forward & 44 & 1343 & CTCACAGGGGGCTTATCTGGGCTTCGGTTCTGGAGGGCCAGGAA 1386 \\
SP-D_1170 & Forward & 56 & 1141 & GATCTGCAGTTGTGAGTTCCTTTTGCAATCGCTGTAGGTCATTGTGCAACCTGCTG 1196 \\
SP-D_1370 & Forward & 50 & 1341 & TTGTGAATATCAGTGGCAGGTTTCCAGAACGCAGGTGGGGATAAGAGTGA 1390 \\
\hline
\end{tabular}

${ }^{a}$ Number of nucleotides (Nt) in target sequence. ${ }^{\mathrm{b}}$ Underlined CG denotes the target $\mathrm{CpG}$.

5 cycles of $95^{\circ} \mathrm{C}$ for $30 \mathrm{sec}, 50^{\circ} \mathrm{C}$ for $1 \mathrm{~min}$, and $72^{\circ} \mathrm{C}$ for $1 \mathrm{~min}$, then $30 \mathrm{cycles}$ of $95^{\circ} \mathrm{C}$ for $30 \mathrm{sec}, 55^{\circ} \mathrm{C}$ for $1 \mathrm{~min}$, and $72^{\circ} \mathrm{C}$ for $1 \mathrm{~min}$, and a final extension at $72^{\circ} \mathrm{C}$ for $4 \mathrm{~min}$ (all primer sequences are listed in Table III). The PCR products were digested with HinfI for SP-A1_1468 and NlaIII for SP-D_1170 according to the manufacturer's instructions (NE Biolabs, Maine). The digested PCR products were separated on $8 \%$ PAGE. The methylated and unmethylated Cs were distinguished by size differences on the gel.

BeadArray ${ }^{T M}$ technology and methylation data analysis. A total of $1536 \mathrm{CpG}$ sites in 371 genes were previously studied and the SP CpG sites were part of this initial data set. Thus, the BeadArray for methylation detection and methylation data analysis, as well as cluster analysis, $\beta$-value, and P-value, were the same as described previously (25).

Bisulfite sequencing. The methylation status of three $\mathrm{CpG}$ sites was examined by bisulfite sequencing. The detailed procedure was described previously (25). The primers used are listed in Table III.

Constructs of SP-A1 alleles $6 A^{3}$ and $6 A^{4}$, and SP-A2 alleles $1 A^{1}$ and $1 A^{5}$. A 1.3-kb PCR fragment of human SP-A 5 flanking and 5'-UTR region (-227-1078) was cloned into pGEM ${ }^{\circledR}$-T vector (Promega, Madison, WI) from 2 individuals with genotype of $6 A^{4} 6 A^{4} / 1 A^{5} 1 A^{5}$, and $6 A^{3} 6 A^{3} / 1 A^{1} 1 A^{1}$. Then the fragments containing variant $6 \mathrm{~A}^{3}, 6 \mathrm{~A}^{4}, 1 \mathrm{~A}^{1}$, or $1 \mathrm{~A}^{5}$ were subcloned into the plasmid pcDNA3 and fused with the reporter gene luciferase (LUC). The insert DNA sequence was verified by sequencing.

Analysis of gene expression of the SP-A1 and SP-D genes. Normal lung tissues were pulverized, and total RNA was extracted from tissue powder with Qiagen RNeasy mini kit (Qiagen Inc. Valencia, CA) according to the manufacturer's instructions. One $\mu \mathrm{g}$ of total RNA was used for RT (reverse transcription) with reverse transcriptase (Invitrogen, San Diego,
CA). One $\mu 1$ of RT products was used as a template for PCR amplification.

For SP-D, the entire coding region was amplified by HotStop PCR with primers 825 and 826 from $1 \mathrm{ul}$ of RT, and the PCR product was separated on $3.5 \%$ PAGE. For control, GAPDH mRNA was amplified by Hot-Stop PCR with primers 1490 and 1491, and the PCR products were separated on 7\% PAGE. For SP-A, PCR amplification was with primers 32A (in 5'UTR) and 68A (3'UTR), and then with nested primers 941 and 943 in Hot-Stop PCR. Primer 941 contains a mismatched nucleotide to convert the variation of SP-A1 and SP-A2 into a $B s m I$ RFLP. The final PCR products were digested with BsmI and separated on $8 \%$ PAGE. The upper band (not cut by the enzyme) is from the SP-A2 mRNA, and the lower band (cut by the enzyme) is from the SP-A1 mRNA (28).

Hot-Stop PCR. For semi-quantitative analysis, SP-A1, SP-D, and GAPDH were amplified by Hot-Stop PCR. Before the last cycle of PCR, the ${ }^{32} \mathrm{P}$-labeled primers 943 (for SP-A1), 825 (for SP-D), and 1490 (for GAPDH) were added, and run for one PCR cycle. The PCR products were separated on PAGE as described above. The gel was dried, and exposed to Phosphorimager screen (Molecular Dynamics, Sunnydale, $\mathrm{CA}$ ) for $2 \mathrm{~h}$ at room temperature. The bands on the films were quantified by using ImageQuant software V 5.2.

\section{Results}

DNA methylation profiling of SPs in lung cancer (adenocarcinoma and SCC). In our previous study (25), a panel of $1536 \mathrm{CpG}$ sites of 371 genes were measured in lung adenocarcinoma and non-cancerous lung tissue samples. A panel of $55 \mathrm{CpG}$ sites identified were associated with lung adenocarcinoma. Here, we report methylation profiling of 11 CpG sites of the surfactant protein genes SP-A1 ( $n=3)$, SP-B $(n=3)$, SP-C $(n=3)$, and SP-D $(n=2)$.

A total of 90 samples were studied, 23 adenocarcinoma tissues and 23 matched non-cancerous tissues, and 22 SCC 


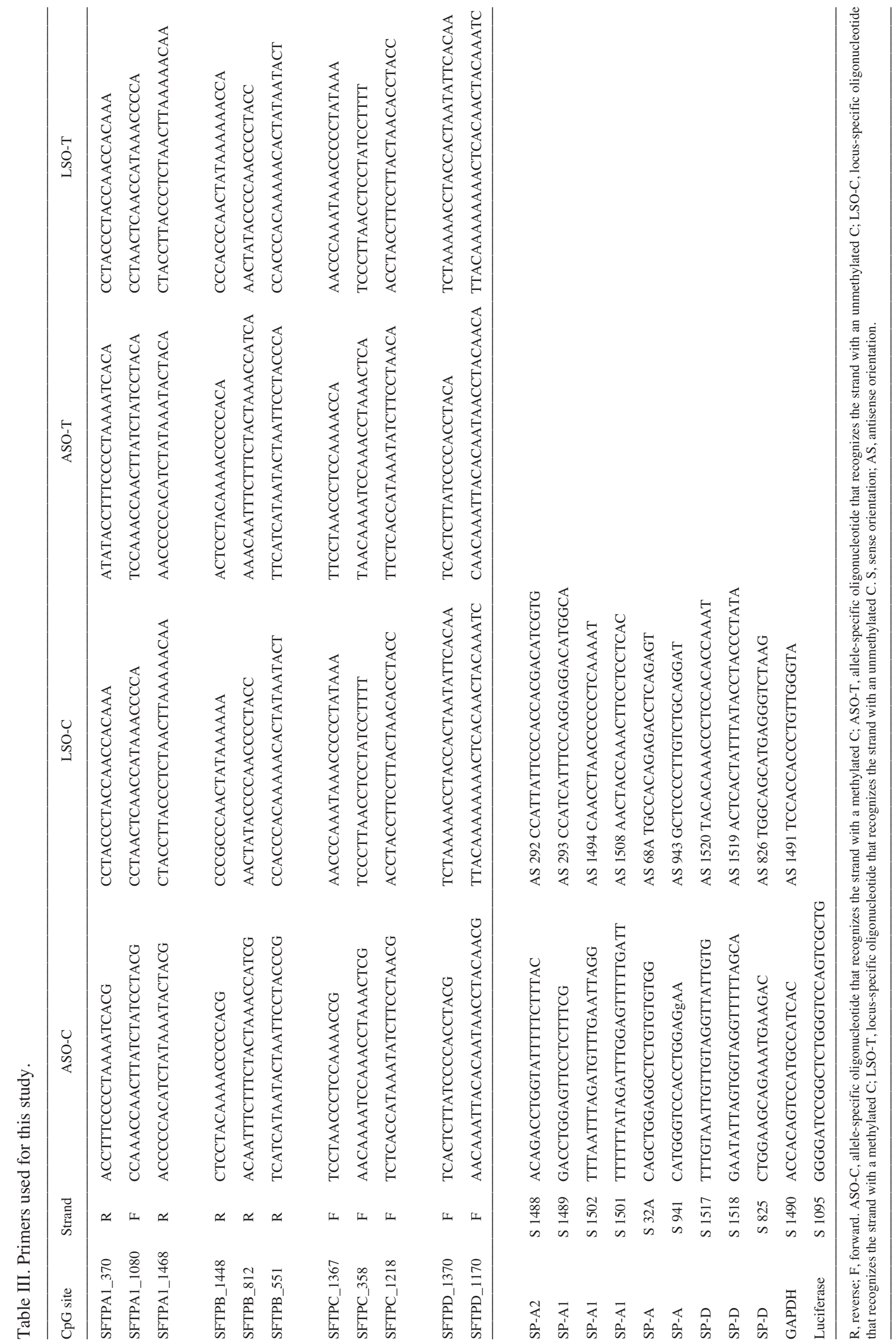




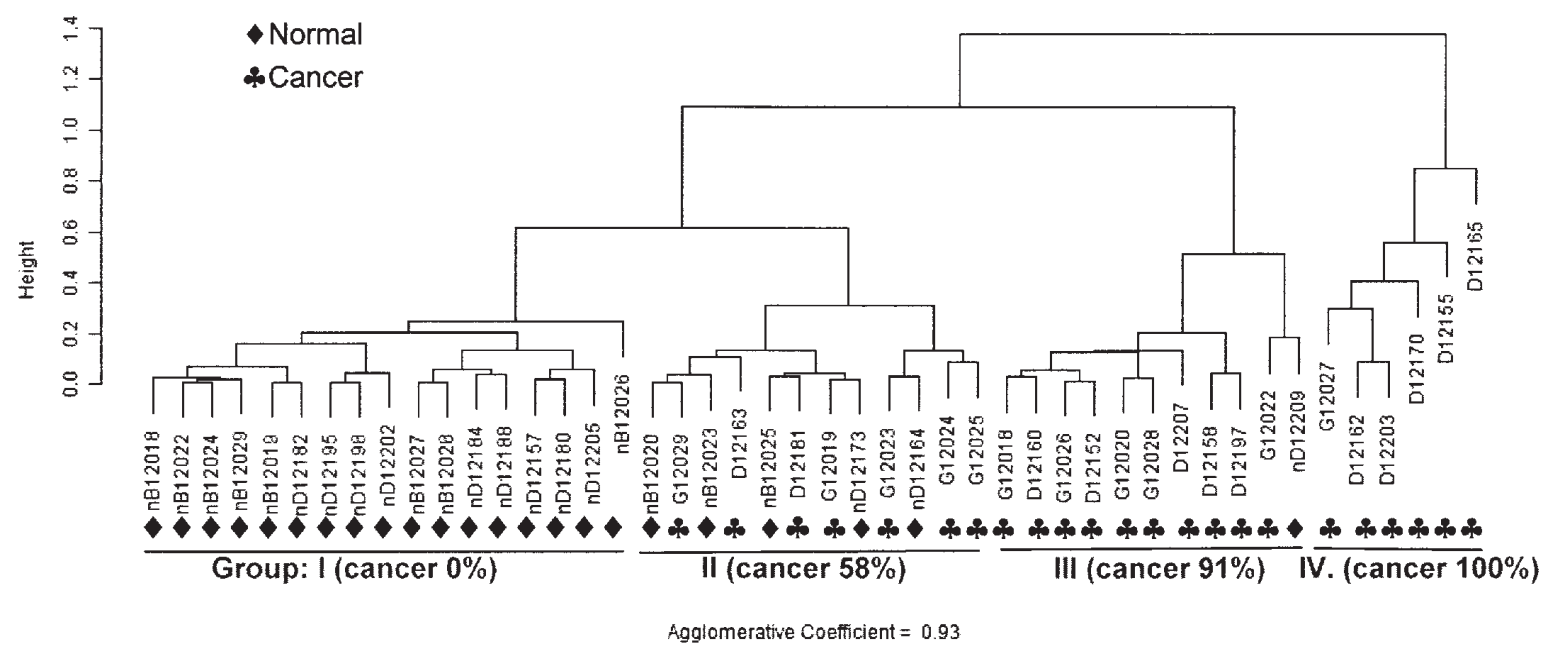

Figure 1. Methylation profiling of surfactant proteins (SPs) in lung adenocarcinoma. Cluster analysis of lung adenocarcinoma with the 11 CpGs of the four SPs. Groups I, II, III, and IV are marked by lines under each cluster. The normal and cancerous tissues are indicated by $\bullet$ and $\bullet$, respectively. In the sample identification, $\mathrm{nB}$ denotes non-cancerous tissues from the training set, $\mathrm{G}$ denotes cancerous tissues from the training set, $\mathrm{nD}$ denotes non-cancerous tissues from the testing set, and D denotes cancerous tissues from the testing set. Height is the distance between sub-clusters, and this is a measure of divergence of their methylation profiles.

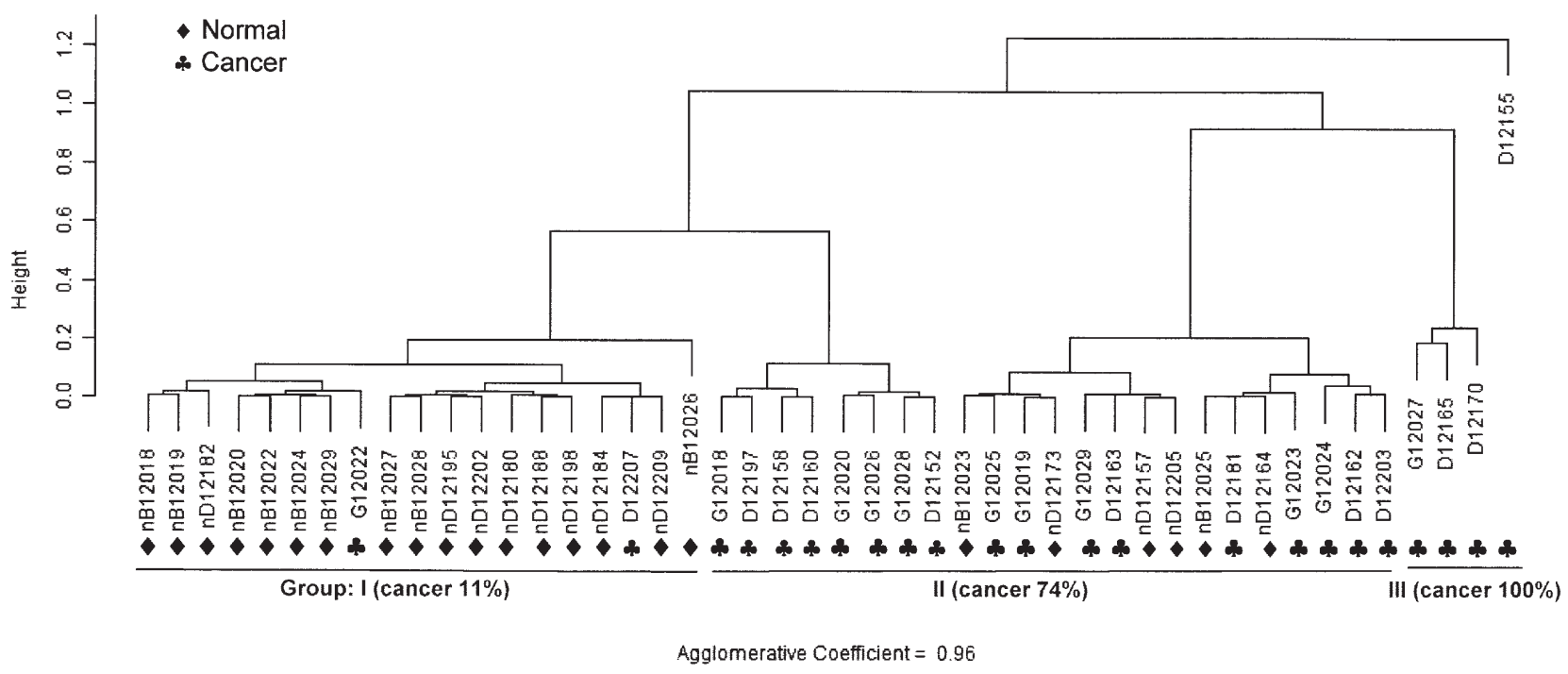

Figure 2. Methylation profiling of surfactant proteins (SPs) in lung squamous cell carcinoma. Cluster analysis of 11 CpGs of SPs in lung squamous cell carcinoma. Groups I, II, and III are marked by lines under each cluster. The normal and cancer tissues are indicated by and $\bullet$, respectively. In the sample $\mathrm{ID}, \mathrm{nB}$ denotes non-cancerous tissues from the training set, $\mathrm{G}$ denotes cancerous tissues from the training set, $\mathrm{nD}$ denotes non-cancerous tissues from the testing set, and $\mathrm{D}$ denotes cancerous tissues from the testing set.

tissues and 22 matched non-cancerous tissues. Each cancerous tissue and matched normal tissue were from the same patient. The DNA methylation profiling was assessed by cluster analysis, where a matrix of co-efficient between calculated methylation signals was computed. Agglomerative nesting was applied using the Agnes function in the $\mathrm{R}$ package with Ward's methods and connection-based distance to cluster. For example, the methylation profiling pattern of the $11 \mathrm{CpG}$ markers of SPs for all 46 samples from patients with adenocarcinoma including the training set and the testing set is shown in Fig. 1.

The cluster analysis of these 46 samples revealed 4 groups as indicated below each cluster group. Group I had 17 samples, and none of them were cancerous tissue. Group II had
12 samples, 5 of them were non-cancerous tissue (42\%), and 7 of them were cancerous (58\%). Group III had 11 samples, only 1 sample was non-cancerous $(9 \%)$ and 10 were cancerous (91\%). Group IV had 6 samples, all of them were cancerous tissue. These results indicate that the methylation pattern of the 11 SP CpGs is associated with lung adenocarcinoma. The overall level of methylation for the markers tested decreases as one moves from group I to group IV. As information related to the clinical status of the subjects from which this tissue was obtained is limited, we could not analyze them further based on clinical outcome of cancer, such as the stage of cancer, or other related information, such as smoking or other known environmental risk factors for the development of lung cancer. The pattern also indicates that the association 
A

\begin{tabular}{c|c|c|c|c}
\hline \multirow{2}{*}{ CpG } & \multicolumn{2}{|c|}{$\begin{array}{c}\text { Bisulfite sequencing } \\
\text { (ratio) }\end{array}$} & \multicolumn{2}{c}{$\begin{array}{c}\text { Micro-Array } \\
\text { (ratio) }\end{array}$} \\
\cline { 2 - 5 } & Normal & Cancer & Normal & Cancer \\
\hline SP-A1_1080 & 0.750 & 0.250 & 0.783 & 0.508 \\
SP-A1_370 & 0.750 & 0.250 & 0.883 & 0.589 \\
SP-C_1367 & 0.909 & 0.333 & 0.797 & 0.240 \\
\hline
\end{tabular}

B

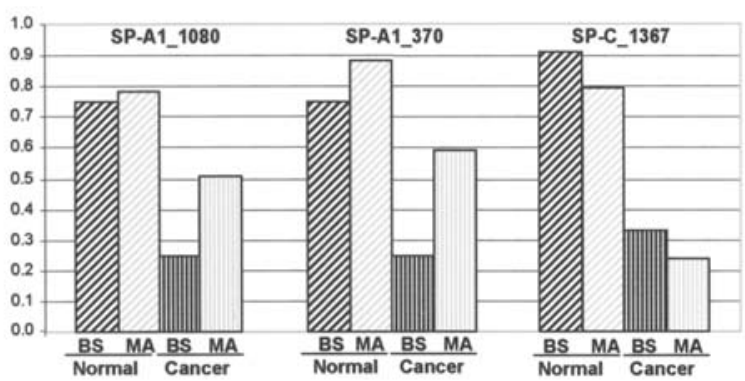

Figure 3. Comparison of methylation data between micro-array-based and bisulfite sequencing methods. A depicts the ratio of the methylated allele to the total of both the methylated and unmethylated alleles in the bisulfite sequencing and micro-array methods. In the bisulfite method, the number indicates the ratio of the clones with the methylated allele to the total sequenced clones with both methylated and unmethylated alleles. In the micro-array method the number indicates the ratio of the fluorescence of the methylated allele to both methylated and unmethylated alleles. These data are shown graphically in B. BS, bisulfite sequencing; MA, micro-array.
Table IV. CpG methylation in SP genes in lung adenocarcinoma and SCC.

\begin{tabular}{lllllll}
\hline & & \multicolumn{2}{c}{ Adenocarcinoma } & & \multicolumn{2}{c}{ SCC } \\
Gene & \multicolumn{1}{c}{ CpG } & Training set & Test set & & Training set & Test set \\
\hline SP-A1 & SP-A1_1080 & $0.003772^{\mathrm{a}}$ & $0.001044^{\mathrm{a}}$ & & $0.027614^{\mathrm{a}}$ & $0.028113^{\mathrm{a}}$ \\
& SP-A1_1468 & 0.001118 & 0.205912 & & 0.293768 & 0.125708 \\
& SP-A1_370 & $0.001602^{\mathrm{a}}$ & $0.003246^{\mathrm{a}}$ & & $0.000513^{\mathrm{a}}$ & $0.069560^{\mathrm{a}}$ \\
SP-B & SP-B_1448 & 0.016894 & 0.021244 & & 0.259249 & 0.680931 \\
& SP-B_551 & 0.234252 & 0.462130 & & 0.414916 & 0.493534 \\
& SP-B_812 & 0.001539 & 0.038327 & & 0.096916 & 0.488472 \\
SP-C & SP-C_1218 & 0.121618 & 0.154626 & & 0.689672 & 0.078421 \\
& SP-C_1367 & 0.066326 & 0.014051 & & 0.454018 & 0.260439 \\
& SP-C_358 & 0.005642 & 0.493035 & & 0.086892 & 0.546864 \\
SP-D & SP-D_1170 & $0.016158^{\mathrm{a}}$ & $0.022731^{\mathrm{a}}$ & & $0.002156^{\mathrm{a}}$ & $0.009680^{\mathrm{a}}$ \\
& SP-D_1370 & $0.009490^{\mathrm{a}}$ & $0.001428^{\mathrm{a}}$ & & $0.003399^{\mathrm{a}}$ & $0.001465^{\mathrm{a}}$ \\
\hline
\end{tabular}

The number presented is the P-value (cancer vs. non-cancerous) determined by t-test for 2 sample sets for each type of cancer. The samples of adenocarcinoma (training set and test set), and squamous cell carcinoma (training set and test set) are described in the Materials and methods. ${ }^{a}$ Significant differences observed with training sets and test sets in both types of cancer.

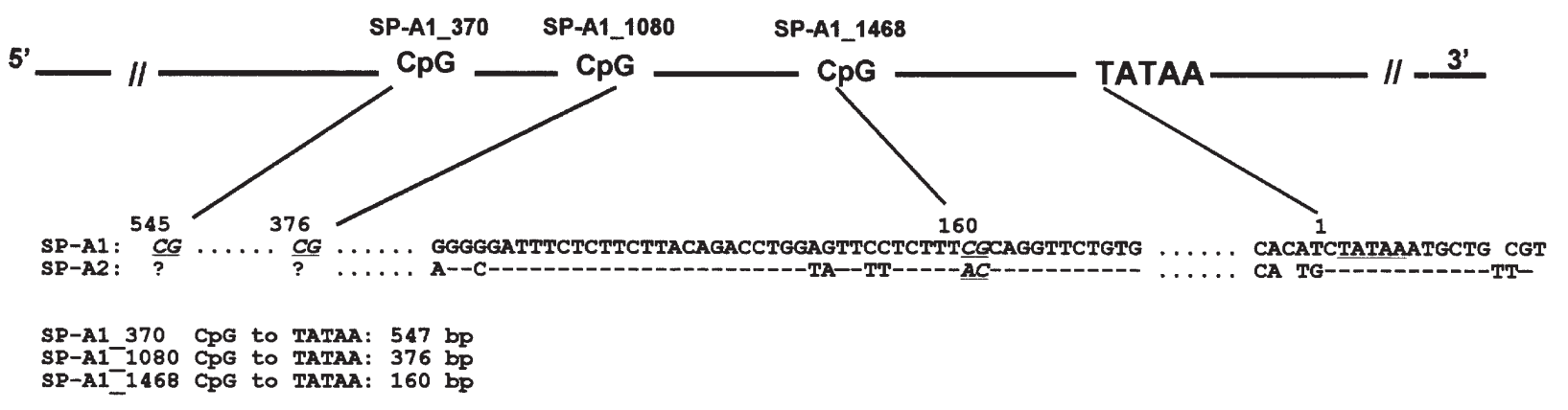

Figure 4. Sequence comparison of CpG sites between SP-A1 and SP-A2 genes. SP-A2 gene sequence flanking CpG SP-A1_1468 site is from our unpublished data. Nucleotide numbers are from 0 (first T in TATAA box) to upstream 160, 376, and 545 according to SP-A1 DNA sequence. The distance between each CpG site and TATAA is shown on the top.

is probably a quantitative trait, as opposed to a dichotomous yes or no explanation.

Clustering analysis of the lung SCC patient samples with the 11 SP markers demonstrated a similar pattern to that observed in adenocarcinoma, but the distinction of normal tissues was not as tightly clustered as that for adenocarcinoma. For example, group I had 19 samples, 17 of them were noncancerous tissue $(89 \%)$ and 2 of them were cancerous tissue $(11 \%)$. Group II had 23 samples, 6 of them were non-cancerous tissue $(26 \%)$ and 17 of them were cancerous (74\%). Group III had 4 samples, all of them were cancerous tissue $(100 \%)$ (Fig. 2).

Identification of candidate methylation markers of surfactant protein genes for lung cancer. We further compared the methylation levels of each $\mathrm{CpG}$ site in cancerous vs noncancerous tissues for all samples, and analyzed the data by t-test. A total of 45 cancerous tissues (23 adenocarcinoma and 22 SCC) and 45 matched non-cancerous tissues were analyzed. The data shown in Table IV indicate that from the 11 CpG markers analyzed, 4 CpG sites (SP-A1_1080, SPA1_370, SP-D_1170, and SP-D_1370) were significantly different between cancerous and non-cancerous samples in both adenocarcinoma and SCC cohorts, and in both the German group and the American group. These results indicate that these $4 \mathrm{CpG}$ markers could be used as potential biomarkers for diagnosis of lung cancer.

Validation of micro-array methylation data by bisulfite sequencing. To validate our micro-array data, we used bisulfite sequencing methods (29) to analyze 3 CpG sites, SP-A1_1170, SP-A1_370, and SP-C_1367. DNA from two normal lung samples and four adenocarcinoma samples were converted with bisulfite and amplified by PCR. PCR fragments were 
A

S: $1489(\mathrm{SP}-\mathrm{A} 1)$

AS : 293 (SP-A1)

SP-A1: ACAGACCTGGAGTTCCTCTTTCGCAgGTTCTGTGCTCCCCTCAAGgGTCCTTTGAGA... TATAAA ... (Exon 2) TGCCATGTCCTCCTGGAAATGATGG

SP-A2:

S: $1488(\mathrm{SP}-\mathrm{A} 2)$

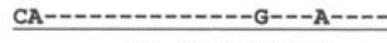

AS : $292(\mathrm{SP}-\mathrm{A} 2)$

B

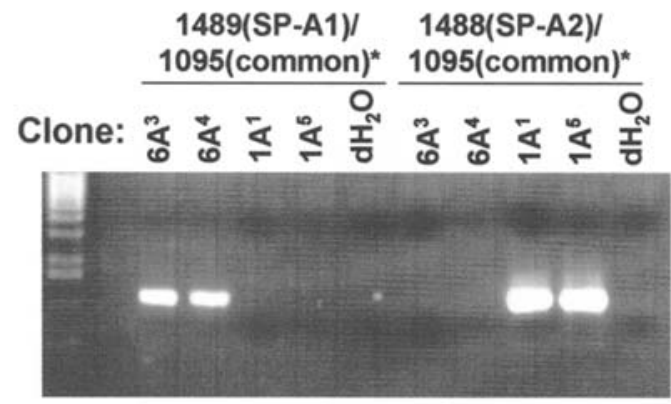

Gene: $\overline{\text { SP-A1 }} \overline{\text { SP-A2 }} \overline{\text { SP-A1 SP-A2 }}$

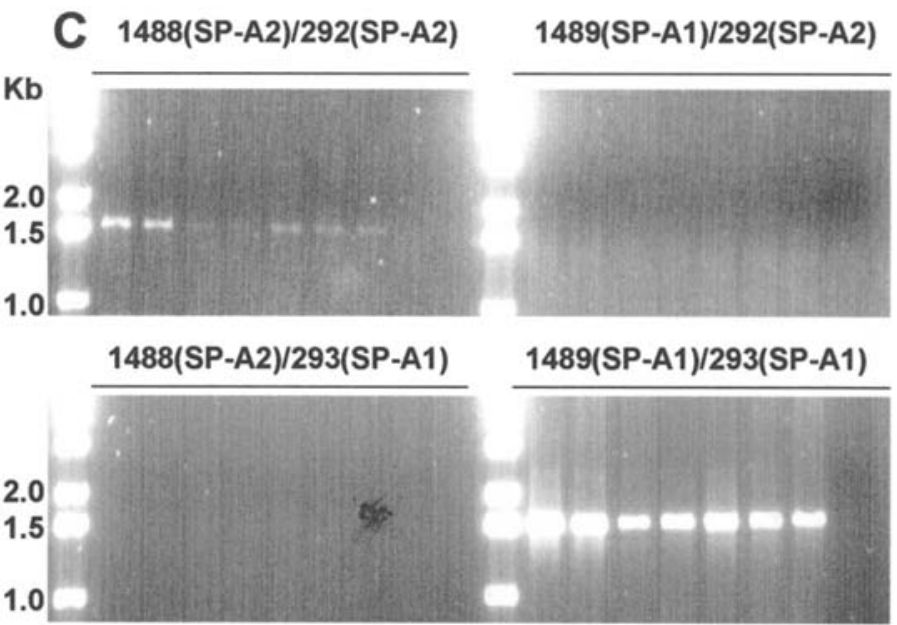

$\begin{array}{llllllllllllllllll}M & 1 & 2 & 3 & 4 & 5 & 6 & 7 & C & M & 1 & 2 & 3 & 4 & 5 & 6 & 7 & C\end{array}$

Figure 5. Gene-specific PCR amplification of SP-A1 and SP-A2. A depicts the sequence, gene location (upstream from TATA or flanking region; exon 2), and orientation (S, sense; AS, antisense) of gene-specific primers, 1488, 1489, 292, and 293 used for PCR (B and C). B depicts PCR amplification of genomic DNA clones of SP-A1 variants $6 \mathrm{~A}^{3}$ and $6 \mathrm{~A}^{4}$, and of SP-A2 variants $1 \mathrm{~A}^{1}$ and $1 \mathrm{~A}^{5}$. ${ }^{*}$ Primer 1095 is a luciferase gene-specific sequence present in the constructs. $\mathrm{C}$ depicts PCR amplification from 7 individual human lung DNAs (\#1 to \# 7): C, PCR control with d $\mathrm{H}_{2} \mathrm{O}$ only; $\mathrm{M}, 1-\mathrm{kb}$ DNA ladder.

cloned, and twelve clones from each cloned fragment were sequenced. The ratio of the methylated allele to the total methylated and unmethylated alleles was calculated. For the micro-array method, the ratio of methylation was calculated by the proportion of the fluorescent signal of the methylated allele to the total of both the methylated and unmethylated alleles (Fig. 3A). The differences between cancerous and non-cancerous tissues are similar in both data sets from the two different methods (Fig. 3B), indicating that the results of the bisulfite sequencing methods could validate those of the micro-array.

The SP-A1_1468 CpG denotes an SP-Al gene-specific DNA methylation site. The 5'-flanking sequence is available for SP-A1 (ENSG00000122852), but not for SP-A2 (ENSG00000182314). However, we have limited the 5' flanking sequence for the SP-A2 gene that includes the CpG SP-A1_1468 (Fig. 4). By sequence comparison, we found that the $\mathrm{CpG}$ island SP-A1_1468 does not exist in SP-A2, where instead of the CG present in SP-A1, an AC is present in SP-A2 (Fig. 5A).

The specificity of CG for the SP-A1 gene was further examined in SP-A1 recombinant clones. These clones that were generated for other ongoing studies contained luciferase as a reporter gene, and were characterized as SP-A1 variants, $6 \mathrm{~A}^{3}$ and $6 \mathrm{~A}^{4}$, and SP-A2 variants, $1 \mathrm{~A}^{1}$ and $1 \mathrm{~A}^{5}$. If the SP-A1 sequence has a CG and SP-A2 has an AC, the 1488 and 1489 primers should be gene-specific for SP-A2 and SP-A1, respectively. As shown in Fig. 5B, with PCR primer 1489 (SP-A1) and a common primer 1095 (based on the sequence of the reporter gene luciferase), only DNAs from the SP-A1 clones were amplified, while with primers 1488 (SP-A2) and
1095, only DNAs from the SP-A2 clones were amplified. Therefore, in the SP-A1 clones the sequence is CG, and in the SP-A2 clones the sequence is AC.

To confirm that the above sequence does not represent a single polymorphism in individual genomes but a genespecific polymorphism, we performed gene-specific PCR amplification in 7 individual genomic DNAs. The primers used were SP-A1-specific primers 293 and 1489, and SP-A2specific primers 292 and 1488. The results of gene-specific PCR from the 7 individual genomic DNAs are shown in Fig. 5C. Only the SP-A1 (1489/293) and SP-A2 (1488/292) primer pairs, but not other combinations of primers, could produce PCR products at the expected size. PCR amplification with primer pairs $1488 / 293$ or 1489/292 resulted, as expected, in no detectable PCR products. The results described indicate that the CpG SP-A1_1468 site is present only in the SP-A1 gene and not in the SP-A2 gene.

\section{Association of $C p G$ methylation with gene expression} a) Association of CpG SP-D_1170 methylation with SP-D gene expression. SP-D_1170 is located at $142 \mathrm{nt}$ upstream of the TATAA box, and its methylation status is significantly associated with lung cancer (Table IV). In 3 samples of lung tissue obtained from individuals that were free from any lung disease, we observed different methylation patterns of the SP-D_1170 site (Fig. 6). In lung 30, this CpG was minimally (if at all) methylated whereas both methylated and unmethylated sites were clearly present in lungs 26 and 48. The majority of this $\mathrm{CpG}$ was unmethylated in lung 48, but lung 26 showed a similar level of methylated and unmethylated $\mathrm{CpG}$ (Fig. 6A). The Hot-Stop PCR results showed that the SP-D transcript 
A

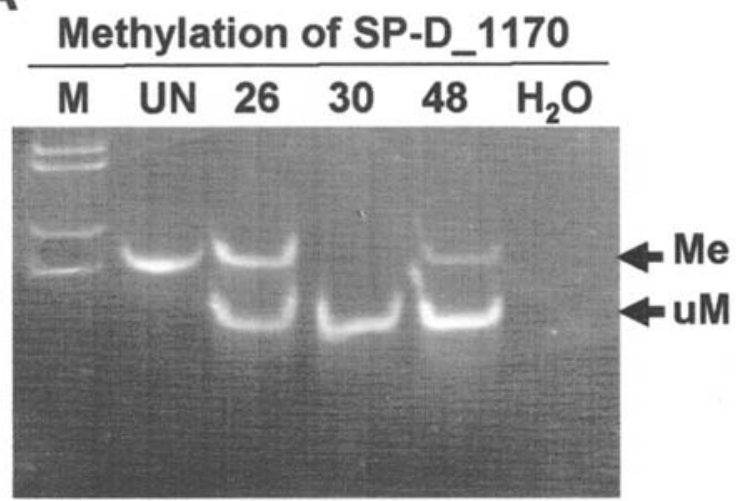

C

\section{Methylation of SP-A1_1468

$\begin{array}{lllll}M & 30 & 44 & 45 & \mathrm{UN} \mathrm{H}_{2} \mathrm{O}\end{array}$

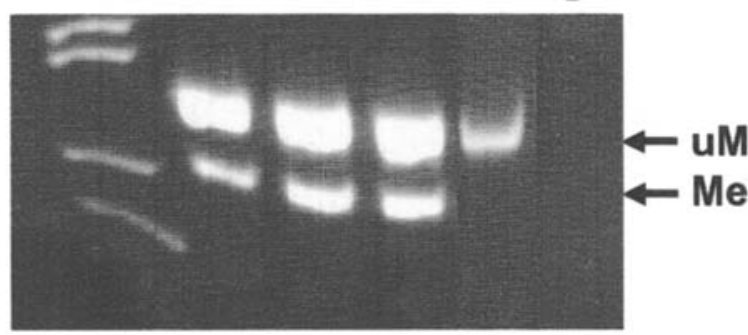

B

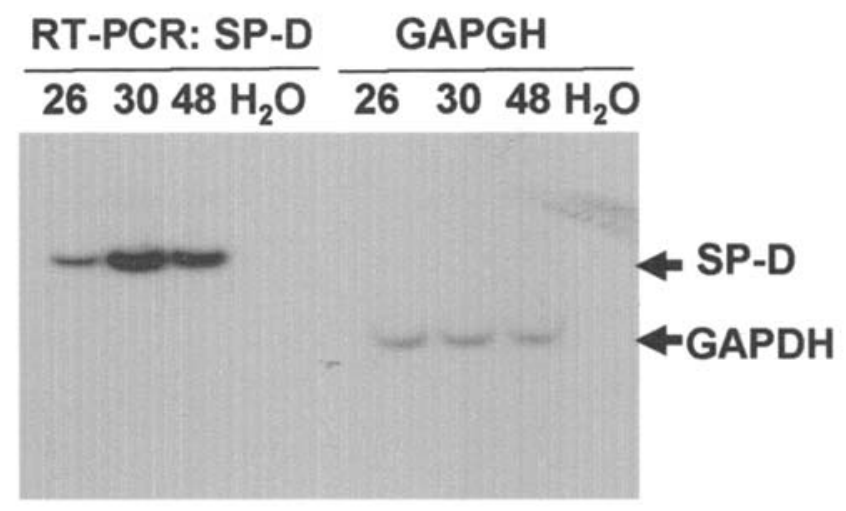

D RT-PCR

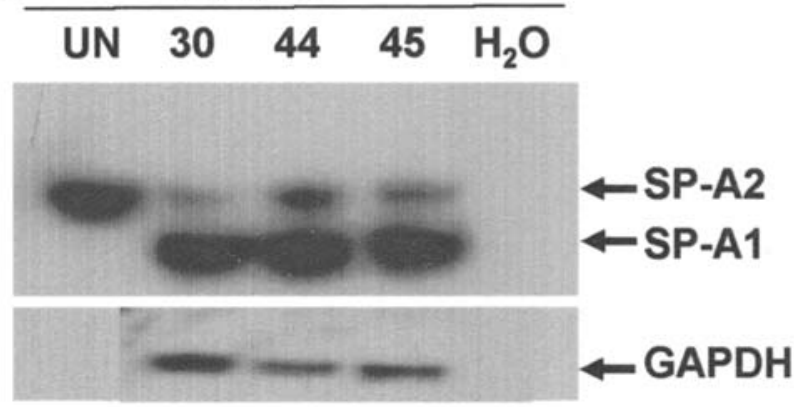

Figure 6. Association of DNA methylation of CpGs SP-D_1170 and SP-A1_1468 with gene expression of the SP-D and SP-A1 in human normal lung tissues. A depicts the DNA methylation analysis of SP-D_1170. Genomic DNAs from three normal human lungs 26, 30 , and 48 were converted by bisulfite and used as templates for PCR amplification with primers 1517/1520, and then nested with primers 1518/1519. The final PCR products were digested with or without (UN) NlaIII. Arrows indicate unmethylated (uM) and methylated (Me) CpG forms. B depicts the analysis of SP-D and GAPDH (control) gene expression. One $\mu 1$ of RT was used for PCR amplification with primers 825 and 826 (SP-D) and 1490 and 1491 (for GAPDH) by Hot-Stop PCR. The PCR products were separated on 3.5\% PAGE. Arrows indicate the PCR products representing SP-D and GAPDH mRNA. C depicts the DNA methylation analysis of SPA1_1468. The genomic DNAs from normal human lung 30, 44, and 46 were converted by bisulfite and used as templates for PCR amplification with primers 1494/1502, and then with nested primers 1501/1508. The final PCR products were digested with or without (UN) HinfI. Arrows indicate unmethylated (uM) and methylated (Me) CpG forms. D depicts the analysis of SP-A1 and SP-A2 gene expression. One $\mu 1$ of RT was used for PCR amplification with primers 32A and 68A. The PCR products were nested with primers 941 and 943 . The final PCR products were digested with or without (UN) BsmI, and then separated on $8 \%$ PAGE. Arrows indicate the PCR products representing SP-A1 and SP-A2 mRNA.

content in sample 30 was the highest, followed by sample 48 and then sample 26 (Fig. 6B). These data show that lungs 26 and 48 with a high level of methylated SP-D_1170 exhibited a low amount of SP-D mRNA, while lung 30 with a high level of unmethylated SP-D_1170 exhibited a higher amount of SP-D mRNA, indicating that DNA methylation of SPD_1170 may inhibit SP-D transcription.

b) Association of SP-A1_1468 CpG methylation and SP-A gene expression. The SP-A1_1468 CpG is located at $160 \mathrm{nt}$ upstream of the TATAA box. To study the methylation status of this CpG island, we developed a simple PCR-based cRFLP method. This method enables analysis of the methylation status of the SP-A1_1468 site in a small scale. Towards this goal, we used 3 samples of lung tissue obtained from individuals that were free from any lung disease, and exhibited different methylation patterns at SP-A1_1468 (Fig. 6C). The methylation content in lung 30 was lower than that in lungs 44 and 45 (lower band, Fig. 6C). To study whether the methylation status of the SP-A1_1468 CpG site has an impact on SP-A1 expression, we compared the relative amount of SP-A1 mRNA to SP-A2 mRNA in these lungs. We used RT-PCR to amplify
SP-A1 and SP-A2 mRNAs, and then distinguished SP-A1 products from SP-A2 by cRFLP analysis, as described in the Materials and methods. PCR primers, 32A, 68A, 941, and 943 used in RT-PCR are common to both SP-A1 and SP-A2 gene sequences. Thus, both SP-A1 and SP-A2 genes should be similarly amplified in the two rounds of PCR. Then the PCR products were digested with BsmI, where PCR products from SP-A1 are cut by $B s m I$, but SP-A2 products are not and thus the SP-A2 products remain at the same position. Fig. 6D indicates that the ratio of SP-A1 to SP-A2 in sample $30(=7.9)$ is higher than the ratio in samples $44(=2.5)$ and $45(=3.6)$. Moreover, Fig. 6C and 6D together indicate that the lower level of gene expression of SP-A1 in lungs 44 and 45 (Fig. 6D) may be the result of the higher level of DNA methylation of SP-A1 at the CpG site SP-A1_1468 (Fig. 6C).

As noted in Fig. 5A, the SP-A1_1468 CpG site is absent in the SP-A2 DNA sequence. Differences in the methylation patterns between SP-A1 and SP-A2 genes are likely to be contributed, at least in part, by the SP-A1_1468 CpG island. Under this scenario, the difference in the ratio of SP-A1 and SP-A2 among samples could reflect changes of SP-A1 expression. However, if there are methylation differences in 
other $\mathrm{CpG}$ sites and/or mechanisms other than methylation are involved in gene-specific regulation, a change in SP-A1 and SP-A2 mRNA content may or may not be detected by methylation of the CpG SP-A1_1468.

\section{Discussion}

In the present study, we investigated whether the DNA methylation state of $11 \mathrm{CpG}$ sites of surfactant protein-A1, -B, $-\mathrm{C}$, and $-\mathrm{D}$ are associated with lung cancer. Four $\mathrm{CpG}$ sites were found to significantly associate with lung cancer. Two of these were from SP-A1 and two were from SP-D. Moreover, the level of methylation, for two out of $11 \mathrm{CpGs}$ studied, appeared, in normal lung tissue, to correlate with SP-A1 and SP-D gene expression. The present data together with the genetic association of surfactant protein genes with lung cancer $(24,31,32)$ indicate that surfactant proteins may contribute to lung cancer genetically and epigenetically.

All species but primates have a single SP-A gene. An SP-A gene duplication occurred with the appearance of primates (16). In humans, the SP-A locus consists of two functional genes, SP-A1 and SP-A2, found in opposite transcriptional orientation, and a pseudogene (43). Evidence indicates that the two SP-A genes differ in many ways, such as sequence variations in nucleotides that may or may not change the encoded amino acid, in 5'UTR splicing, in levels of mRNA and protein in vivo, and in the thermal stability of the SP-A1 and SP-A2 proteins. These, collectively, may alter protein properties and/or SP-A function $(16,44,45)$. It is generally accepted that SP-A1 and SP-A2 are differentially regulated at various levels $(16,46)$. The finding in the present study, that the SP-A1 gene-specific CpG SP-A1_1468 site is regulated by DNA methylation, and that this is associated with differences in SP-A mRNA content, provides yet another potential mechanism for study regarding differential regulation of SPA1 and SP-A2. In other systems $(10,47,48)$, regulation of DNA methylation or demethylation processes are subject to a large number of environmental stimuli, and these processes have been shown to play a role in allele-specific expression. Differential allele-specific expression of SP-A and SP-D has been previously observed in the rat $(49,50)$. These data together support the possibility that a methylation/demethylation mechanism is operative in the regulation of SP-A and SP-D gene expression.

Both SP-A and SP-D play important roles in lung host defense and the regulation of inflammatory processes $(15,30)$. Inflammation may be a consequence of a specific immune reaction to the tumor. Tumor invasion can be heavily influenced by the presence of an inflammatory infiltrate. Evidence from both animal models and cancer patients indicates that the immune system is able to recognize foreign (i.e. different viral) or endogenous tumor-associated antigens (such as human carcinoembryonic antigen) (13). It is estimated that inflammation contributes to the development of at least $15 \%$ of all cancer (14). Association of lung inflammatory diseases with an increased risk of the development of lung cancer has been well demonstrated by epidemiological studies (12). Individuals with chronic obstructive pulmonary disease (COPD) exhibiting chronic inflammation are at an elevated risk for subsequent development of lung cancer (33). Chronic bronchitis (34) and asthma (35) also heighten the risk of lung cancer. Many factors can cause lung inflammation, including a variety of environmental antigens and pollutants. To meet the challenge, host defense molecules in the lung, such as SP-A1 and SP-D $(15,30)$, as well as other genes $(14,36)$, may play a critical role in the regulation of inflammatory processes and consequently in the pathogenesis of certain subtypes of lung cancer.

Dendritic cells (DCs) constitute one of the many types of cells proposed to participate in tumor inflammation. DCs in lung tumor infiltrates are often defective in their ability to stimulate T-cells (37). SP-A and SP-D affect DC maturation and gene expression in opposite directions. SP-A negatively impacts maturation of DCs (38) whereas SP-D appears to enhance expression of the co-stimulatory molecule CD 86 and enhance antigen presentation (39). It is possible that under normal conditions, the health status of the tissue is partly maintained via a delicate balance between inhibitory and stimulatory activities of SP-A and SP-D. We speculate that in lung cancer this fine balance is disturbed with increased levels of SP-A due to its hypomethylation status. In fact increased levels of SP-A mRNA have been observed in peripheral blood from patients with lung carcinomas (40). Moreover, it has been shown that SP-A-producing cells may generate a number of lung cancers (41).

Although hypomethylation of SP-D was also shown to occur in lung cancer (present study), currently it is unknown whether, in the tumor microenvironment, the change in the relative methylation level of SP-A and SP-D is similar or whether these changes result in significant differences in the relative SP-A and SP-D protein content. Should the latter be the case, the putative delicate balance between inhibitory and stimulatory activities of SP-A and SP-D on DCs, discussed above, may become deranged, and this in turn may compromise proper activation of adaptive tumor immunity and failure to eliminate tumor cells. It should also be noted that not all lung tumors have been identified as SP-A positive and this may be explained by a recent finding where SP-A gene deletion was observed in stage I non-small cell lung cancer (42).

In summary, we have previously reported a panel of 55 adenocarcinoma-specific methylation markers that was identified from the screening of $1536 \mathrm{CpG}$ sites in 371 genes (25). Here, we studied $11 \mathrm{CpG}$ sites specific to the SP genes and observed that four of these, SP-A1_370 and SP-A1_1080, and SP-D_1170 and SP-D_1370, were significantly hypomethylated in lung cancer samples of adenocarcinoma and squamous cell carcinoma in the studied groups of German and American cohorts; and two (SP-D_1170; SP-A1_1468) CpGs, in normal human lung tissues, not only exhibited differential methylation content but samples with lower levels of methylation were associated with higher content of mRNA, indicating a correlation between CpG DNA methylation and mRNA content. We speculate that SP CpGs contribute to lung cancer via derangement in the regulation of their expression. Whether all significant $\mathrm{CpG}$ methylation markers (25, present study) associate with gene expression remains to be determined. However, collectively these markers could, via high throughput technologies, be used as screening markers for lung cancer. Thus, the combination of current multiplex high-throughput technologies and further non-invasive sampling methods may 
provide a powerful tool for both lung cancer diagnosis as well as monitoring the response to clinical treatment. Furthermore, they may offer the possibility for early detection through non-invasive screening of subjects at risk for lung cancer.

\section{Acknowledgements}

This study is supported by NIH R37 HL34788 to J.F., NIH R44 CA097851-02 to J.-B.F., American Lung Association Research Award RG-066-N to Z.L., and a research grant to N.J.T. from the Jane B. Barsumian Trust, The Pennsylvania State University College of Medicine. The authors gratefully acknowledge the Gift of Life Donor Program (Philadelphia, PA) and the generosity of the organ donor families for allowing the organs that are not suitable for transplantation to be utilized to advance the understanding of human disease.

\section{References}

1. Egger G, Liang G, Aparicio A and Jones PA: Epigenetics in human disease and prospects for epigenetic therapy. Nature 429: 457-463, 2004.

2. Laird PW and Jaenisch R: The role of DNA methylation in cancer genetics and epigenetics. Annu Rev Genet 30: 441-464, 1996.

3. Baylin SB and Herman JG: DNA hypermethylation in tumorigenesis: epigenetics joins genetics. Trends Genet 16: 168-174, 2000.

4. Jones PA and Baylin SB: The fundamental role of epigenetic events in cancer. Nat Rev Genet 3: 415-428, 2002.

5. Jones PA and Laird PW: Cancer epigenetics comes of age. Nat Genet 21: 163-167, 1999.

6. Herman JG and Baylin SB: Promoter-region hypermethylation and gene silencing in human cancer. Curr Top Microbiol Immunol 249: 35-54, 2000.

7. Esteller M, Avizienyte E, Corn PG, et al: Epigenetic inactivation of LKB1 in primary tumors associated with the Peutz-Jeghers syndrome. Oncogene 19: 164-168, 2000.

8. Herman JG: Epigenetics in lung cancer: focus on progression and early lesions. Chest 125 (suppl 5): 119S-122S, 2004.

9. Tsou JA, Hagen JA, Carpenter CL and Laird-Offringa IA: DNA methylation analysis: a powerful new tool for lung cancer diagnosis. Oncogene 21: 5450-5461, 2002.

10. Li S, Hursting SD, Davis BJ, McLachlan JA and Barrett JC: Environmental exposure, DNA methylation, and gene regulation: lessons from diethylstilbesterol-induced cancers. Ann NY Acad Sci 983: 161-169, 2003.

11. Moore LE, Huang WY, Chung J and Hayes RB: Epidemiologic considerations to assess altered DNA methylation from environmental exposures in cancer. Ann NY Acad Sci 983: 181-196, 2003.

12. Malkinson AM: Role of inflammation in mouse lung tumorigenesis: a review. Exp Lung Res 31: 57-82, 2005.

13. Brigati C, Noonan DM, Albini A and Benelli R: Tumors and inflammatory infiltrates: friends or foes? Clin Exp Metastasis 19: 247-258, 2002.

14. Marx J: Cancer research. Inflammation and cancer: the link grows stronger. Science 306: 966-968, 2004.

15. Floros J and Phelps DS: Pulmonary surfactant. In: Anesthesia: Biologic Foundations. Yaksh TL, Lynch C III, Zapol WM, Maze M, Biebuck J and Saidman LJ (eds). Lippincott-Raven, pp1259-1279, 1997.

16. Floros J, Wang $\mathrm{G}$ and Lin Z: Genetic diversity of human SP-A, a molecule with innate host defense and surfactant-related function; characteristics, primary function, and significance. Curr Pharmacogenomics 3: 87-95, 2005.

17. Lin Z, deMello DE, Wallot $\mathrm{M}$ and Floros J: An SP-B gene mutation responsible for SP-B deficiency in fatal congenital alveolar proteinosis: evidence for a mutation hotspot in exon 4 . Mol Genet Metab 64: 25-35, 1998.

18. Clark JC, Wert SE, Bachurski CJ, et al: Targeted disruption of the surfactant protein B gene disrupts surfactant homeostasis, causing respiratory failure in newborn mice. Proc Natl Acad Sci USA 92: 7794-7798, 1995.

19. Floros J and Kala P: Surfactant proteins: molecular genetics of neonatal pulmonary diseases. Annu Rev Physiol 60: 365-384, 1998.
20. Floros J and Fan R: Surfactant protein A and B genetic variants and respiratory distress syndrome: allele interactions. Biol Neonate 80 (suppl 1): 22-25, 2001.

21. Floros J and Lin Z: Genetic variability of surfactant protein-B and respiratory distress diseases. Medscape Resp Care 13: 1-6, 1999.

22. Haataja R, Ramet M, Marttila R and Hallman M: Surfactant proteins $\mathrm{A}$ and $\mathrm{B}$ as interactive genetic determinants of neonatal respiratory distress syndrome. Hum Mol Genet 9: 2751-2760, 2000.

23. deMello DE and Lin Z: Pulmonary alveolar proteinosis: a review. Pediatr Pathol Mol Med 20: 413-432, 2001.

24. Seifart C, Seifart U, Plagens A, Wolf M and von Wichert P: Surfactant protein B gene variations enhance susceptibility to squamous cell carcinoma of the lung in German patients. Br J Cancer 87: 212-217, 2002.

25. Bibikova M, Lin Z, Zhou L, et al: High-throughput DNA methylation profiling using universal bead arrays. Genome Res 16: 383-393, 2006.

26. Fan JB, Oliphant A, Shen R, et al: Highly parallel SNP genotyping. Cold Spring Harb Symp Quant Biol 68: 69-78, 2003.

27. Galinsky VL: Automatic registration of microarray images. II. Hexagonal grid. Bioinformatics 19: 1832-1836, 2003.

28. Lin Z, deMello D, Phelps DS, Koltun WA, Page M and Floros J: Both human SP-A1 and Sp-A2 genes are expressed in small and large intestine. Pediatr Pathol Mol Med 20: 367-386, 2001.

29. Frommer M, McDonald LE, Millar DS, et al: A genomic sequencing protocol that yields a positive display of 5-methylcytosine residues in individual DNA strands. Proc Natl Acad Sci USA 89: 1827-1831, 1992.

30. Phelps DS: Surfactant regulation of host defense function in the lung: a question of balance. Pediatr Pathol Mol Med 20: 269-292, 2001 .

31. Saitoh H, Shimura S, Fushimi T, Okayama H and Shirato K: Detection of surfactant protein-A gene transcript in the cells from pleural effusion for the diagnosis of lung adenocarcinoma. Am J Med 103: 400-404, 1997.

32. Seifart C, Lin HM, Seifart U, et al: Rare SP-A alleles and the SP-A1-6A(4) allele associate with risk for lung carcinoma. Clin Gen 68: 128-136, 2005.

33. Cohen BH, Ball WC Jr, Brashears S, et al: Risk factors in chronic obstructive pulmonary disease (COPD). Am J Epidemiol 105: 223-232, 1977.

34. Mayne ST, Buenconsejo J and Janerich DT: Previous lung disease and risk of lung cancer among men and women nonsmokers. Am J Epidemiol 149: 13-20, 1999.

35. Wu AH, Fontham ET, Reynolds P, et al: Previous lung disease and risk of lung cancer among lifetime nonsmoking women in the United States. Am J Epidemiol 141: 1023-1032, 1995.

36. Coussens LM and Werb Z: Inflammation and cancer. Nature 420: 860-867, 2002

37. Katsenelson NS, Shurin GV, Bykovskaia SN, Shogan J and Shurin MR: Human small cell lung carcinoma and carcinoid tumor regulate dendritic cell maturation and function. Mod Pathol 14: 40-45, 2001.

38. Brinker KG, Garner $\mathrm{H}$ and Wright JR: Surfactant protein A modulates the differentiation of murine bone marrow-derived dendritic cells. Am J Physiol Lung Cell Mol Physiol 284: L232-L241, 2003.

39. Brinker KG, Martin E, Borron P, et al: Surfactant protein D enhances bacterial antigen presentation by bone marrow-derived dendritic cells. Am J Physiol Lung Cell Mol Physiol 281: L1453-L1463, 2001.

40. Yamamoto O, Takahashi H, Hirasawa M, et al: Surfactant protein gene expressions for detection of lung carcinoma cells in peripheral blood. Resp Med 99: 1164-1174, 2005.

41. Uzaslan E, Stuempel T, Ebsen M, et al: Surfactant protein A detection in primary pulmonary adenocarcinoma without bronchioloalveolar pattern. Respiration 72: 249-253, 2005.

42. Jiang F, Caraway NP, Nebiyou Bekele B, et al: Surfactant protein A gene deletion and prognostics for patients with stage I non-small cell lung cancer. Clin Cancer Res 11: 5417-5424, 2005.

43. Hoover RR and Floros J: Organization of the human SP-A and SP-D loci at 10q22-q23. Physical and radiation hybrid mapping reveal gene order and orientation. Am J Respir Cell Mol Biol 18: 353-362, 1998.

44. Wang G, Bates-Kenney SR, Tao JQ, Phelps DS and Floros J: Differences in biochemical properties and in biological function between human SP-A1 and SP-A2 variants, and the impact of ozone-induced oxidation. Biochemistry 43: 4227-4239, 2004. 
45. Mikerov AN, Umstead TM, Huang W, Liu W, Phelps DS and Floros J: SP-A1 and SP-A2 variants differentially enhance association of Pseudomonas aeruginosa with rat alveolar macrophages. Am J Physiol Lung Cell Mol Physiol 288: L150-L158, 2005.

46. Wang G, Guo X and Floros J: Differences in the translation efficiency and mRNA stability mediated by 5'-UTR splice variants of human SP-A1 and SP-A2 genes. Am J Physiol Lung Cell Mol Physiol 289: L497-L508, 2005.

47. Cooney CA, Dave AA and Wolff GL: Maternal methyl supplements in mice affect epigenetic variation and DNA methylation of offspring. J Nutr 132 (suppl 8): 2393S-2400S, 2002 .
48. Jaenisch R and Bird A: Epigenetic regulation of gene expression: how the genome integrates intrinsic and environmental signals. Nat Genet 33 (suppl): 245-254, 2003.

49. Lin $\mathrm{Z}$ and Floros J: Heterogeneous allele expression of pulmonary SP-D gene in rat large intestine and other tissues. Physiol Genomics 11: 235-243, 2002.

50. Lin Z, Wang Y, Zhu K and Floros J: Differential allele expression of host defense genes, pulmonary surfactant protein-A and osteopontin, in rat. Mol Immunol 41: 1155-1165, 2004. 